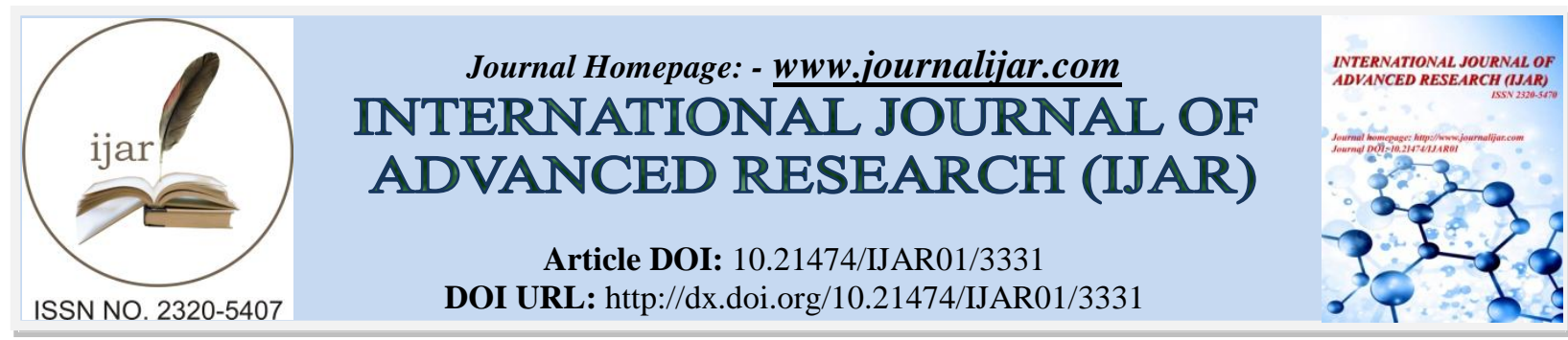

RESEARCH ARTICLE

\title{
FAULT DETECTION AND EVENT PREDICTION IN NETWORKS USING FEATURE MATRIX.
}

\section{Farrukh Arslan.}

Distributed Multimedia Systems Lab, Department of Electrical and Computer Engineering, School of Engineering, Purdue University, West Lafayette, IN, 47907, USA.

\section{Manuscript Info}

Manuscript History

Received: 20 December 2016

Final Accepted: 28 January 2017

Published: February 2017

Key words:prediction; artificial intelligence; network; fault

\section{Abstract}

A data features selection and organization scheme is presented for future event predictions using historical event data. Most of the reported event prediction methods use data features in the form of time series. The proposed approach uses simple data feature statistics. First an event is defined based on historical event data features for all discrete time instances, then all events are ordered chronologically and divided into $\mathrm{N}$ time- windows with an overlapping interval. The probabilities (relative frequencies) for occurrence of all sequences of two events are calculated for each time-window and stored in a 3D input feature matrix. A prediction technique is trained using probabilities from one time-window to learn to predict probabilities for the next time-window. Once the prediction technique is iteratively trained for all $\mathrm{N}$ time-windows of the training data, it is used to predict future probabilities. The accuracy is calculated by comparing with the known test data probabilities. The proposed approach is tested for fault prediction in telecommunication networks using various Artificial Intelligence Techniques.

Copy Right, IJAR, 2017,. All rights reserved.

\section{Introduction:-}

This paper presents a scheme of feature selection and organization of historical event data, useful for future event predictions. The future event is a personal event in the life of a person or nation or trend in economic markets or climate [1]. The accuracy of the prediction depends on the quality and amount of historical event data available and if some kind of correlation is present in the data. The main premise of this paper is that good prediction accuracy depends on finding good patterns in historical event data and good patterns are better found when proper event data features are selected and properly organized. Therefore the same historical event data can be organized and looked at differently resulting in different prediction accuracies [2].

The first step is to select problem domain specific data features that uniquely define distinct events. These unique events are chronologically organized into overlapping time- windows with each time-window representing a state of the historical event data within the bounds of the time-window. The probabilities (relative frequencies) of occurrence of all possible sequences of two events within each time-window are calculated and stored in a three dimensional (3D) feature input

Matrix such that two dimension (time-window) contains probability of occurrence of sequence of events and the third dimension is chronological organization of time-windows. Therefore probabilities within a time-window 
represent local event correlations and changes from window to window represent global event correlations. Please note that we use the term probability for relative frequency.

The historical event data used, for this paper, is the historical alarm log of a telecommunication system. The historical alarm $\log$ is first preprocessed to remove duplicates and insignificant alarms. Then based on alarm properties, unique events are identified and the probabilities of occurrence of all possible sequences of two events are calculated per overlapping time-window and stored in a 3D matrix. The 3D matrix is used as input to train a prediction technique, so that the prediction technique learn to produce probabilities of occurrences of all sequences of 2 events for the next window for any given window in the training set. The trained prediction technique is used to predict the probabilities of all event sequences for a future window. The set of predicted probabilities in the future time-window are compared with a set of pre-calculated probabilities of the test data to calculate prediction accuracies. False positives and negatives are considered for the calculation of these accuracies. The proposed approach of using the 3D matrix is unique, novel and is a simple method that provides accurate future fault predictions. The prediction results of 4 prediction techniques: Artificial Neural Network (ANN), Support Vector Machine (SVM), Kalman Filter (KF) and Hidden Markov Model (HMM). Please note that although the problem domain discussed contains fault events in telecommunication systems, the proposed 3D input feature matrix can be calculated for any problem domain and discussion presented is applicable to historical event data from any problem domain.

The rest of the paper is organized in 4 sections: The first covers some reported prediction techniques relevant to this paper, the second describes the proposed 3D matrix, the third presents prediction results for the 4 prediction techniques and the last section is the conclusion.

\section{Event Prediction Techniques:-}

Here some event prediction works are discussed. One such technique is used to predict traffic while also incorporating weather parameters [3]. Stationary Wavelet Transform (SWT) is used in conjunction with ANN. SWT is used to separate out approximation and details components from traffic and precipitation time series data. ANN is used to predict these components and then recombined using an inverse SWT. This SWT-ANN model is applied on real traffic and weather data for better accuracy than ANN alone, due to the feature processing of the data by the SWT.

Another technique predicts rain rates, to alleviate wireless signal attenuation, using conditional Gaussian Distribution of rain rate [4]. The historical data consists of rain rate and rain attenuation measurements with samples taken per second. Conditional occurrences of rain rate values are modeled, in a time series, using a Gaussian Distribution. The data (rain rate) at one instance is conditional on data (rain rate) in the previous instant. This model is successfully used to predict rain rates in 2008 from data of 2005-2007.

A multivariate reconstructed phase space (MRPS) is proposed to extend the univariate reconstructed phase space [5]. The premise is that multivariate data sequences are better than univariate data sequences for finding temporal patterns in dynamic data. The argument is that dynamic data contains sequences that are anomalously generated by hidden states and a univariate method will miss out on local trends and shifts. A real life application, of this method, is to detect causes of sludge bulking treatment plants.

Another application is to predict blood glucose concentration using meal information in addition to regular glucose readings [6]. Although the history of glucose concentration values can predict future glucose levels, the glucose levels also depend on food and medicine intake and physical exercise intensity. Therefore capturing of relations between glucose levels and meal intake, in a time series, can provide better predictions of future glucose levels. An ANN model predicts sum of 2 values, one is glucose prediction by a linear predictor and second is estimation of error in the first prediction. The error estimation is done on the basis of current prediction error, trend of prediction error, actual glucose measurement, glycemic trend and carbohydrate intake. The addition of food intake data has made glucose prediction much more accurate especially after and before a meal.

The work described in [7] is closer to our problem domain anomaly (fault) detection in operational networks such as customer care logs, system events, network alarms etc. All of these contain a lot of information about system health and performance. A time series prediction is an ideal choice for detection of anomalies in the system in operation. In the reported Tiresias system, heavy hitters (faults) are detected in real time using data hierarchy 
present in operational logs. The dataset used is acquired from a broadband provider and consists of customer care logs and Setup box crash event logs. The input time series contains classification information and date-time stamp of a particular dataset. The classification is done using a hierarchical tree where nodes identify dataset weight of a category and time unit in which that data has been recorded. A sliding window concept is used to partition the data in time units. Arrival of new data adds more windows and older windows may be deleted to keep data from increasing too much. A separate seasonal analysis is done, using Fourier Transform and Wavelet Transform, to automatically choose seasonal parameters. The seasonal forecasting is done using Holt-Winters forecasting model.

The approach described by C. Xu et. al. is similar to the proposed approach [8]. Event sequence semantics are used in a Support Vector Regression (SVR) based predictive model. A semantic vector is defined that contains factors that affect the probability of occurrence of an event and weights for these factors. The time wise ordered events are partitioned in to Basic Windows (BWs) with interval T between BWs. For each BW, a standardized semantic vector is formed based on the sequence of events in BW and factors affecting the sequence of events. Now this se-mantic vector is used in the predictive model rather than the events. A hierarchical clustering algorithm is presented that uses a "Confidence" calculation (a measure of likelihood of one event following another event) instead of probability of occurrence of one event after another. The hierarchical clustering algorithm groups semantic vectors of similar semantics such that center of each group replaces all original semantic vectors and the frequency of the center is the total frequencies of all se-mantic vectors in the group. An event data dependent similarity threshold values decide number of groups. Furthermore a measure of "semantic distance" between event sequences is defined for the clustering algorithm. Different number and size of groups are formed using the similarity threshold and the semantic distance. A SVR based predictive model is built using the groups formed by the clustering algorithm. Experimental results are presented to show the general capability to predict events. A few papers are related to the domain of fault prediction using various prediction techniques [9] [10]. Here one approach uses hybrid fuzzy systems for stock price predictions [11].

The research covered above is a small sample of various novel and unique techniques reported for event prediction. As with the above event prediction techniques, most of the reported techniques use highly complicated algorithms and data processing techniques. Although various ways are suggested on how to augment the historical dataset to improve prediction accuracy, a significant effort is needed in order to evaluate the benefits of proper data organization and features. The work presented in this current paper suggests a simpler way of event data feature calculation and organization, for high prediction accuracies.

\section{The Proposed 3d Data Feature Matrix:-}

In current event prediction techniques, the historical event data is considered in terms of one or more time series with data points at a certain time as one event e.g. weather or stock prices and other related factors on a particular day. Alternatively, rather than using time series, it is proposed to use some simple statistics available in the historical event data for future event prediction. For example, instead of using univariate (e.g. the price of a single stock) or mutivariate time series (e.g. number of related and unrelated stock prices along with other relevant associated parameters), one can also define some unique state of the stocks as an event and then calculate the proposed feature input matrix. Another example is that of predicting the spread of a contagious disease by defining a unique event using number of affected patients and other information about the patients i.e. relationships between patients and demographics. Therefore it is proposed that a prediction technique may provide better prediction accuracies if first an appropriate event, based on data features, is defined and then event based statistics are used. In the predictive model, first find local patterns in the parameters of each event based on overlapping time-windows and then use a prediction technique (e.g. ANN) to learn how these parameters change from time- window to time-window. For every historical datum, an appropriate (optimal) time-window and overlap interval size can be found that best captures these local and global trends thus increasing the accuracy of the prediction technique.

The proposed input feature matrix is used to predict future impending faults in a telecommunication system using historical alarm log. Alarm management is an essential part of the FCAPS of the Telecommunication Management Network (TMN) standard and all telecommunication networks provide alarms management in one form or other [12]. These alarms are reported by the network constituent network elements and contain system health information in chronological order. For this paper, historical logs of three months of alarms from a telecommunication company are used. These alarms have been collected by an element management system 
from the transmission unit of an operational telecommunication network. Here some definitions are in order:

1. Feature (or Parameter): is a unique property of an alarm e.g. equipment, severity

2. Unique Event $(U E)$ : is a unique combination of alarm features (parameters) e.g. equipment type, severity, slot and rack no. It should be noted that a $U E$ is not necessarily an alarm of the telecommunication system.

3. Sequence of Unique Events $(S U E)$ : is a consecutive occurrence of two UEs. For example one UE A followed by another $U E \mathrm{~B}$ in time. A and $\mathrm{B}$ can be same or different.

4. Probability of Occurrence of a SUE (POS): is the probability of occurrence of a SUE. POS is basically number of occurrences of an $S U E$ divided by total number of $S U E \mathrm{~s}$

5. Time-window $(T W)$ : is a partition of historical event data by a time interval. A $T W$ is defined by date-time bounds and may contain $m S U E$ s. For example a $T W$ of one month can be from April 1, 2014:00:00 to April 30, 2014:00:00 and contains $S U E_{1}, S U E_{2}, \ldots ., S U E$

6. Overlapping Interval $(O I)$ : is the overlapping between $T W \mathrm{~s}$. There will be $N T W \mathrm{~s}$ where $N$ depends on the size of $T W$ and $O I$. $T W$ is a multiple of $O I$, therefore $T W=O I$ means no overlap between $T W s$ and $T W=2 * O I$ means $50 \%$ overlap

7. Input Feature Matrix $(I F M)$ : is a three dimensional matrix containing $\mathrm{N}$ TWs. IFM is used as an input to a prediction technique e.g. ANN. $I F M=\{T W 1, T W 2, \ldots . T W N$

8. Training Data: is the portion of historical event data used for training of a prediction technique

9. Test Data: is the portion of historical event data used for calculation of false and true positives in prediction accuracies.
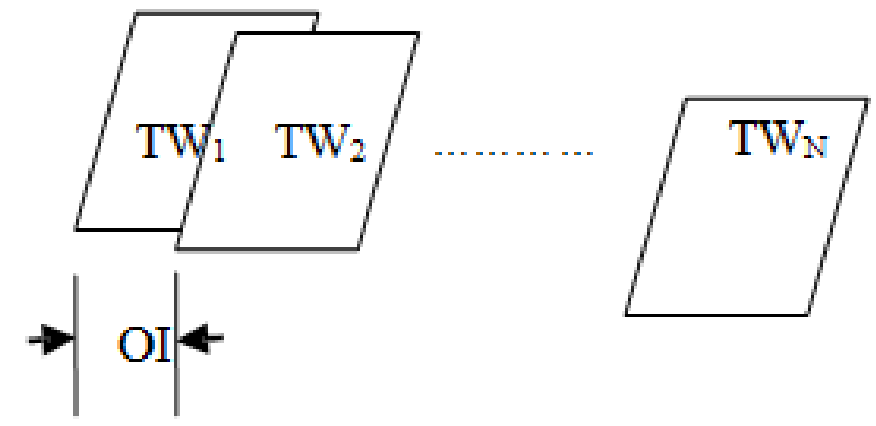

Fig. 1:- N Time-windows (TW) with Overlapping Interval (OI)

Fig. 1 shows that chronologically ordered UEs are divided into $N$ equal size TWs with a predefined $O I$. Larger $T W$ contains more UEs and smaller $O I$ results in more time- windows. The POS for all SUEs are calculated for each $T W$. This calculation of $P O S$, per $T W$, is organized in the form of a two dimensional matrix. This $2 \mathrm{D}$ matrix basically records the probability of occurrence of one $U E$ against another $U E$ (see table I \& II below).

Historical Alarm Data.

\begin{tabular}{|l|l|l|l|l|l|}
\hline Alarm & Alarm & Severity & Probable Case & ALM_IMA_LINK_LCD & Managed \\
\hline 42193 & Link & Major & EMS \\
\hline $\begin{array}{l}\text { Managed } \\
\text { Element }\end{array}$ & Rack & Shelf & Slot & Reason & Date \\
\hline PTP & 1 & 1 & 7 & Loss of Signal & $12 / 09 / 2013: 4: 27$ \\
\hline
\end{tabular}

Fig. 2:- A typical Telecommunication Alarm

The alarm data used is acquired from three months of alarm logs from the transmission network of a telecommunication company. In three months about 45 hundred thousand alarms are generated and take 10 Giga Bytes (text) of disk space. Fig. 1 shows a typical alarm format containing information about date-time of alarm occurrence, network equipment information, severity level and probable cause. The historical alarm log is preprocessed to remove redundant and insignificant alarms. After pre-processing, all $U E$ are found using alarm features and given a $U E$ Identification Number (UEIN).

Table I:- Unique (Alarm) Events partitioned in $N$ TWs. This 3D matrix is used as an input to train a prediction technique. 


\begin{tabular}{|c|c|c|c|c|c|}
\hline $\begin{array}{c}\text { Alarm } \\
\text { Type ID }\end{array}$ & $\begin{array}{l}\text { Severity } \\
\text { Level ID }\end{array}$ & $\begin{array}{l}\text { Probable } \\
\text { Cause ID }\end{array}$ & $\begin{array}{l}\text { Managed } \\
\text { Object ID }\end{array}$ & Alarm Reason & $\begin{array}{l}\text { Unique } \\
\text { Event }\end{array}$ \\
\hline 1 & 1 & 7 & 57 & Unknown & 1 \\
\hline 1 & 1 & 57 & 4251 & Unknown & 2 \\
\hline 1 & 2 & 3 & 2 & Loss Of Signal & 3 \\
\hline 1 & 2 & 3 & 20285 & Loss Of Signal & 4 \\
\hline 1 & 2 & 3 & 45616 & Loss Of Signal & 5 \\
\hline 1 & 2 & 28 & 192 & Loss Of Signal & 6 \\
\hline 2 & 2 & 5 & 46071 & $\begin{array}{c}\text { Equipment } \\
\text { Malfunction }\end{array}$ & 7 \\
\hline 2 & 2 & 174 & 19662 & $\begin{array}{l}\text { Equipment } \\
\text { Malfunction }\end{array}$ & 8 \\
\hline 2 & 2 & 174 & 19663 & $\begin{array}{c}\text { Equipment } \\
\text { Malfunction }\end{array}$ & 9 \\
\hline 3 & 2 & 102 & 19610 & $\begin{array}{c}\text { External IF Device } \\
\text { Problem }\end{array}$ & 10 \\
\hline
\end{tabular}

\section{Prediction Algorithm:-}

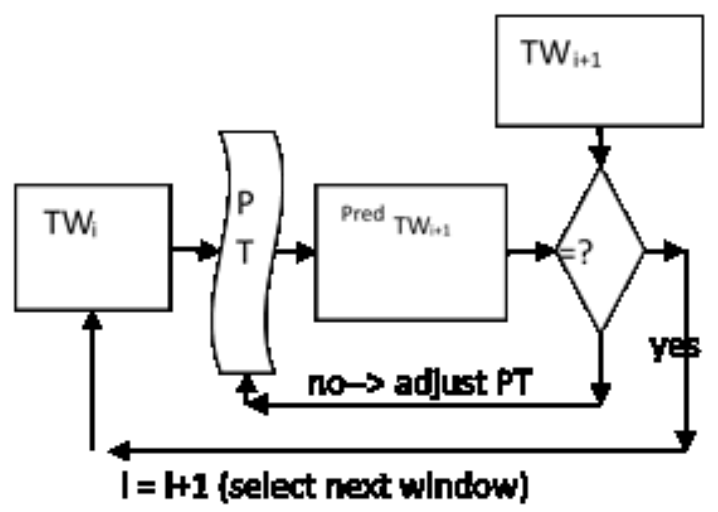

Fig. 3:- shows how a prediction technique $(\mathrm{PT})$ is trained starting with the $i^{\text {th }} \mathrm{TW}$ to predict $P O S$ values in the $i+1^{\text {th }} T W i+1$ The $P O S$ values contained in $T W i$

Table I shows an example of 10 of these UEs for the three months alarm log. The first 5 columns in table I are the alarm features that are used to select the UEs and last column is the index given to a particular $U E$. These preprocessed, chronologically ordered $U E s$ are partition into $T W s$ according to $T W$ and $O I$ size parameters.

Table Ii:- $10 x 10$ Pos Matrix

\begin{tabular}{|c|c|c|c|c|c|c|c|c|c|c|}
\hline & $\mathbf{1}$ & $\mathbf{2}$ & $\mathbf{3}$ & $\mathbf{4}$ & $\mathbf{5}$ & $\mathbf{6}$ & $\mathbf{7}$ & $\mathbf{8}$ & $\mathbf{9}$ & $\mathbf{1 0}$ \\
\hline $\mathbf{1}$ & 0.1 & 0.8 & 0.2 & 0.1 & 0.5 & 0.7 & 0.1 & 0.9 & 0.6 & 0.1 \\
\hline $\mathbf{2}$ & 0 & 0 & 0.2 & 0.3 & 0.4 & 0.6 & 0.7 & 0.4 & 0.1 & 0.8 \\
\hline $\mathbf{3}$ & 0 & 0.6 & 0.6 & 0.1 & 0.7 & 0.9 & 0 & 0.8 & 0 & 0 \\
\hline $\mathbf{4}$ & 0.3 & 0.5 & 0 & 0.8 & 0 & 0.7 & 0 & 0.6 & 0 & 0.6 \\
\hline $\mathbf{5}$ & 0.5 & 0 & 0.8 & 0 & 0 & 0.1 & 0.9 & 0 & 0.1 & 0.8 \\
\hline $\mathbf{6}$ & 0.4 & 0.1 & 0.6 & 0.3 & 0.4 & 0 & 0.8 & 0.3 & 0 & 0 \\
\hline $\mathbf{7}$ & 0.9 & 0.4 & 0.8 & 0 & 0 & 0 & 0.1 & 0 & 0.8 & 0.8 \\
\hline $\mathbf{8}$ & 0.3 & 0.2 & 0.6 & 0.5 & 0.9 & 0.6 & 0.8 & 0.4 & 0.8 & 0.8 \\
\hline $\mathbf{9}$ & 0.5 & 0.4 & 0.6 & 0.7 & 0.4 & 0 & 0.8 & 0 & 06 & 0.6 \\
\hline $\mathbf{1 0}$ & 0.6 & 0.2 & 0 & 0.7 & 0 & 0 & 0.1 & 0 & 0 & 0 \\
\hline
\end{tabular}

Table II shows a 10x10 POS matrix for all the UEs in table I of an $i^{\text {th }} T W$. In this case the value in cell (i, $\mathrm{j}$ ) is the 
probability of occurrence of sequence of $U E j$ happening after $U E i$. For instant $(3,2)=0.2$ means sequence of $U E$ $\mathrm{C}$ happening after $U E \mathrm{~B}$ has a probability of occurrence 0.2 within that $T W$. The probability calculations of occurrence of $S U E$ are done for all $N T W s$ and that forms the proposed 3D data feature matrix. Therefore this 3D matrix contains $U E$ sequence occurrence statistics for the whole historical data.

POS values of PTWi+1. Calculated POS values of $\mathrm{TW} i+1$ are compared with predicted POS values of PredTWi+1. If mean square error (MSE) is acceptable (denoted by ' $=$ ' in the figure) then index is incremented to repeat for the next TW, otherwise the prediction technique parameters are adjusted until MSE is acceptable. For example starting with first set of POS values in $T W_{1}$, the prediction technique is trained to predict values for set of $P O S$ of values in $T W 2$. Now prediction technique is trained to predict set of $P O S$ values in $T W 2$. Similarly prediction technique is iteratively trained for all TWs. The prediction technique training is stopped when MSE from all $T W s$ is below a predefined threshold. Please note that the prediction technique parameter adjustment is incremental from $T W$ to $T W$ and the finally trained prediction technique is based on the local (adjacent $T W s$ ) as well as global ( $T W$ to $T W$ for the whole training period) correlations. Once training is completed, the corresponding trained prediction technique is saved and is ready for prediction of alarm sequences in the future $T W$. A number of experiments are done on the training data to find optimal prediction technique parameters e.g. for ANN these parameters are learning rate, number of hidden layers, number of neurons in each layer and maximum number of epochs. The prediction technique is used to predict $P O S$ values in a future $T W$. All the SUEs with high $P O S$ are checked to see if any contains a $U E$ with severity equal to "Critical". A $U E$ having a severity equal to "Critical" is by definition a fault and must be reported. Furthermore other SUE are also useful for prediction of noncritical UEs and overall health of the system.

\section{Fault Prediction Results:-}

The overall historical alarm data is divided into training dataset and test dataset. The test dataset size depends on the number of TWs (size of overlapping interval OI). The POS values are also calculated for all the TWs of prediction dataset. The trained prediction technique is use to predict $P O S$ values in the prediction dataset and accuracy calculations are done using

The predicted POS values and actual POS values of the prediction dataset. The accuracy calculations are done using the following 2 parameters:

Accuracy:- It is defined as the ratio of correct predictions to the total possible prediction cases.

Accuracy $=($ No. of true positives + No. of true negatives $) /$ Total No. of Unique Events that can be predicted $* 100$

Here the true negative mean the number of UEs that are neither predicted nor are present in original future window. Accuracy provides an idea about how well our system is predicting the UEs. There is another factor which must be taken into account in testing a prediction.

False Positive Rate: It is defined as the ratio of false positive cases to Total number of Negative cases. False Positive Rate $=$ No. of False Positives $/($ No. of True Negatives + No. of False Positives) $* 100$

Note that the denominator is basically the UEs that are absent in the predicted window. False Positive Rate is the measure of penalty due to over prediction. If accuracy of a technique is good but False Positive Rate is too high then it means that $U E s$ are predicted but do not actually occur.

The three months alarm data is divided into training and prediction datasets on a 70:30 (percent) basis. The training datasets are used to train the prediction system using four techniques: Artificial Neural Network (ANN), Support Vector Machine (SVM), Kalman Filter (KF) and Hidden Markov Model (HMM). A number of experiments are performed using all of the four prediction techniques. The prediction technique is trained using the alarm characteristics (POS) of a window as input and the alarm characteristics (POS) of future window as output. Once the prediction system is trained, Accuracy and False Positive Rates are calculated for all the training examples and are averaged to get a mean value.

Now the rest of the $30 \%$ prediction windows are totally unknown to the predictor. A number of prediction experiments are performed and evaluated. The Accuracy and False Positive Rates are averaged over all the 
prediction experiments. This is to evaluate how well the fault prediction system performs for unknown data. That is why some techniques that have performed well for training data, show poor results for unknown data predictions, whereas other prediction techniques that have relatively poor performance for training data, show good unknown data prediction results. Note that this process is done repeatedly for various TW and corresponding OI values.

The optimum OI values for each TW value have also been obtained after thorough experimentations. The detail analysis of these optimal parameters will be reported elsewhere. For different TW and OI, Table III and IV show average Accuracy values and Table V and VI show False Positive Rate values for the training and prediction datasets.

Table Iii:- Percentage Accuracy For The Training Dataset.

\begin{tabular}{|l|l|l|l|l|l|}
\hline TW & \multicolumn{1}{|c|}{ OI } & Prediction Technique & SVM & KF & HMM \\
\hline & & ANN & SVM & 73.14 & 70.5 \\
\hline $4 \mathrm{hrs}$ & $2 \mathrm{hrs}$ & 78.25 & 98.92 & 70.91 & 69.1 \\
\hline $10 \mathrm{hrs}$ & $5 \mathrm{hrs}$ & 76.26 & 98.86 & 65.76 & 56.0 \\
\hline 2 days & 1 day & 69.77 & 98.97 & 59.75 & 51.4 \\
\hline 4 days & 2 days & 67.91 & 99.37 & \multicolumn{2}{l|}{} \\
\hline
\end{tabular}

Table Iv;- Percentage Accuracy For Unknown Prediction Dataset

\begin{tabular}{|c|c|c|c|c|c|}
\hline TW & OI & \multicolumn{4}{|c|}{ Prediction Technique } \\
\hline & & ANN & SVM & KF & HMM \\
\hline $4 \mathrm{hrs}$ & $2 \mathrm{hrs}$ & 54.67 & 66.67 & 93.33 & 54.34 \\
\hline $10 \mathrm{hrs}$ & $5 \mathrm{hrs}$ & 54.67 & 58.67 & 93.67 & 58 \\
\hline 2 days & 1 day & 54.34 & 54.67 & 93.76 & 41.67 \\
\hline 4 days & 2 days & 50.34 & 56.67 & 88 & 48 \\
\hline
\end{tabular}

Table V:- Percentage False Positive Rates For Training Dataset.

\begin{tabular}{|l|l|l|l|l|l|}
\hline TW & \multicolumn{2}{|c|}{ OI } & Prediction Technique & KF & HMM \\
\hline & & ANN & SVM & KN & 4.5 \\
\hline $4 \mathrm{hrs}$ & $2 \mathrm{hrs}$ & 1.826 & 2.04 & 1.45 & 7.78 \\
\hline $10 \mathrm{hrs}$ & $5 \mathrm{hrs}$ & 1.76 & 1.578 & 1.106 & 20.09 \\
\hline days & 1 day & 2.86 & 1.907 & 1.22 & 16.93 \\
\hline
\end{tabular}

Table Vi:- Percentage False Positive Rates For Unknown Prediction Dataset

\begin{tabular}{|c|c|c|c|c|c|}
\hline TW & OI & \multicolumn{4}{|c|}{ Prediction Technique } \\
\hline & & ANN & SVM & KF & HMM \\
\hline $4 \mathrm{hrs}$ & $2 \mathrm{hrs}$ & 31.3 & 36 & 8 & 55.67 \\
\hline $10 \mathrm{hrs}$ & $5 \mathrm{hrs}$ & 46 & 38.67 & 7.34 & 53 \\
\hline 2 days & 1 day & 39.6 & 5.76 & 0.00 & 34 \\
\hline 4 days & 2 days & 46 & 8.67 & 16.34 & 33.34 \\
\hline
\end{tabular}

The above tables (III and IV) show that for training data, SVM clearly shows best predictions for all four window sizes.

But we have to decide which technique outperforms others when it comes down to random and unknown test data windows. Table $\mathrm{V}$ shows that for prediction data, KF is the best prediction technique. Although it has been only the third favorite of the four techniques in case of training data, KF gives best prediction accuracies for various cases of random test data windows. False Positive Rate from table VI also confirms that the lowest False Positive Rate for training as well as prediction datasets is shown by KF, almost for all four window sizes. Next best choice is SVM. The reason behind KF performing best in unknown data case is that KF does not have the tendency to get as much over trained (during the training phase) as other techniques. 
Furthermore from the four tables III, IV, V and VI, the best performance is for a TW $=10$ hours and OI $=5$ hours. Therefore for the given alarm data, it is recommended to use TW of 10 hours and OI of 5 hour. It should be noted that after experiments the optimal values of TW and OI can be found for any historical event data for any problem domain. The same is true for finding out the optimum prediction technique for a problem. Please note that the individual detail of ANN, SVM, KF and HMM parameter tuning and optimization are omitted due to conference paper size constraints and will be reported in an extended version of this paper.

\section{Conclusion:-}

A simple statistical approach, as an alternate to more complex time series based approaches, is presented for future events prediction. After selection of appropriate problem domain based features, Unique Events (UEs) are defined and historical event data is partitioned into overlapping Time- windows (TWs). For each TW, Probabilities of Sequence of Unique Events (POS) values are calculated and stored in a three dimensional (3D) Input Feature Matrix (IFM). The 3D IFM contains local (within a TW) and global (TW to TW) correlations and a prediction technique is better able to predict future events. The proposed event prediction technique is applied to the domain of telecommunication alarms and future faults are predicted using three months of transmission system alarm log of a telecommunication company. Various experiments are done using different TW and OI values for four prediction techniques to find optimal TW and OI values.

Although the results show highly accurate results, we are not claiming that the proposed 3D input matrix will always produce the same level of prediction accuracy for all types of data. Our premise is that the highly accurate results are due to the calculated POS values and then used in the form of the proposed 3D IFM. Intuitively we feel that the proposed 3D input matrix should produce optimal prediction for any data. Although a lot of experimentation is needed with different prediction algorithm and in different problem domains to really validate the proposed approach. Such an effort is currently underway and detailed results will be reported in the future.

\section{Acknowledgment:-}

This work was done when author was working in distributed multimedia system lab at Purdue University. Acknowledgment is due to the director of distributed multimedia systems lab for his guidance and the people for sharing data and resources.

\section{References:-}

1. Editorial 2012.: Election forecasting in neglected democracies: An introduction. International Journal of Forecasting 28: 767-768

2. Kazmi, A. 2011.: Application of Statistical Sampling to Predict Faults from Real Time Alarm Data. 14th International Multitopic Conference 290-295. IEEE Press USA.

3. Dunne, S. and Ghosh, B. 2013.: Weather Adaptive Traffic Prediction Using Neurowavelet Models. IEEE Transactions on Intelligent Transportation Systems 14(1):370-379

4. Das, D. and Maitra, A. 2012.: Time series prediction of rain rate during rain events at a tropical location. IET Microwaves, Antennas \& Propagation 6(15): 1710-1716.

5. Zhang, W. and Feng, X. 2014.: Event Characterization and Prediction Based on Temporal Patterns in Dynamic Data System. IEEE Transactions on Knowledge and Data Engineering 26(1): 144-156.

6. Zecchin, C.; Facchinetti, A.; Sparacino, G.; Nicolao, G. and Cobelli, C. 2012. Neural Net-work Incorporating Meal Information Improves Accuracy of Short-Time Prediction of Glucose Concentration. IEEE Transactions on Biomedical Engineering 59(6):1550-1560

7. Hong, C. and Duffield,N. 2012.: Tiresias: Online Anomaly Detection for Hierarchical Operational Network Data. 32nd IEEE International Conference on Distributed Computing Systems 173-182. IEEE Press USA

8. Xu, C.;Lin, S; Qiao, J.; Yu, G. and Zhang, T.: Research on Event Prediction Algorithm Based on Event Sequence Semantic. Sixth International Conference on Fuzzy Systems and Knowledge Discovery. 2009. IEEE Press USA

9. Xu, Z.; Ji, Y. and Zhou, D.2009:. A New Real-Time Reliability Prediction Method for Dynamic Systems Based on On-Line Fault Prediction. IEEE Transactions on Reliability 58(3): 523-538

10. Szilagyi, P. and Novaczki, S. 2012. An Automatic Detection and Diagnosis Framework for Mobile Communication Systems", IEEE Transactions on Network and Service Management. 9(2):184-197

11. Zarandi, H.; Hadavandi, E. and Turksen, I. 2012. A hybrid fuzzy intelligent agent-based sys-tem for stock price prediction. International Journal of Intelligent Systems 27(11):947-969

12. Recommendation M.3400 (02/00). Telecommunication Management Network (TMN) Management Function. International Telecommunication Union (ITU). 2001. 